\title{
Preparation and Humidity Sensing Properties of Silver Oxide Added Bismuth Iron Molybdate
}

\author{
V. Jeseentharani, J. Dhineshkumar, K. S. Nagaraja, A. Dayalan, B. Jeyaraj* \\ Department of Chemistry, Loyola Institute of Frontier Energy (LIFE), \\ Loyola College, Chennai 600034, India. \\ ${ }^{*}$ Corresponding author. Tel.:+91 44 28178200; fax: +91 4428175566 \\ E-mail addresses: jeseentha@gmail.com; jeyaraj.boniface@gmail.com
}

\begin{abstract}
:
$\mathrm{Bi}_{3} \mathrm{FeMo}_{2} \mathrm{O}_{12}$ (BFM, Bismuth iron molybdate) was prepared by wet chemical method by using metal nitrates and ammonium molybdate as a precursor and the powder was heated up to $700^{\circ} \mathrm{C}$ for $24 \mathrm{~h}$. Silver nitrate was added up to 5 and $10 \mathrm{~mol} \%$ to BFM.compound. BFM: $\mathrm{Ag}_{2} \mathrm{O}_{3}$ composites with mol ratios of 100:0 (BFM-1), 95:5(BFM-2) and 90:10 (BFM-3) were prepared and their humidity sensitivity measurements were studied. The composites were characterized by X-ray diffraction (XRD) and Scanning Electron Microscopy (SEM). The surface area measurement was obtained by the nitrogen adsorption/desorption study by BET method. The sensitivity factor $S_{\mathrm{f}}$ was calculated by the ratio of resistances, $R_{5 \%} / R_{98 \%}$, where $R_{5 \%}$ and $R_{98 \%}$ are the $d c$ resistances at $5 \% \mathrm{RH}$ and $98 \% \mathrm{RH}$ respectively. Response and recovery time were measured by using a degassed glass chamber. The BFM-2 possessed the highest humidity sensitivity of $16798.57 \pm 550$ while the other two composites possessed a sensitivity factor of $5177.4 \pm 180$ and $13914.1 \pm 350$ for BFM-3 and BFM-1, respectively. The surface area of BFM-1, BFM-2 and BFM-3 showed $8.81,20.66$ and $15.79 \mathrm{~m}^{2} / \mathrm{g}$ respectively. The response and recovery time of BFM-2 was 260 and $180 \mathrm{~s}$ respectively.
\end{abstract}

Key words: humidity sensor, BET analysis, Bismuth Iron Molybdate.

\section{Introduction}

The measurement of humidity has received great attention due to the recognized importance of vapour concentration in many areas such as meteorology, medicine, industry and agriculture [1-3]. The relative humidity $(\mathrm{RH})$, which is the ratio of actual vapour pressure to the saturated vapour pressure at a given temperature, is the most frequently used parameter to specify humidity. Metal molybdates are important inorganic materials that have a high application potential in various fields [4]. $\mathrm{Bi}_{3} \mathrm{FeMo}_{2} \mathrm{O}_{12}$, tribismuth(III) iron(III) molybdate $(\mathrm{VI})$, has a frame work structure derived from that of scheelite. The present study deals with the humidity sensing properties of bismuth iron molybdate powder and the effect of silver(III) oxide addition to the bismuth iron molybdate compound.

\section{Experiment and fabrication:}

BFM: $\mathrm{Ag}_{2} \mathrm{O}_{3}$ composites with mol ratios of 100:0 (BFM-1), 95:5(BFM-2) and 90:10 (BFM-3) were prepared and their humidity sensitivity measurements were studied. The bismuth iron molybdate, $\left(\mathrm{Bi}_{3} \mathrm{FeMo}_{2} \mathrm{O}_{12}, \mathrm{BFM}-1\right)$ can be prepared by a process of co-precipitation method [5-7]. Bismuth iron molybdate (BFM-1) was ground for $3 \mathrm{~h}$ in acetone for the homogeneous powder. To this BFM-1, 5 (BFM2) and 10 (BFM-3) mol \% of silver nitrate $\left(\mathrm{AgNO}_{3}\right)$ was added and ground for $2 \mathrm{~h}$. The samples were then made in the form of cylindrical pellets of dimension $13 \mathrm{~mm}$ diameter and $2-3 \mathrm{~mm}$ thickness using a hydraulic press at a pressure of $400 \mathrm{MPa}$. The pellets were then sintered at $700^{\circ} \mathrm{C}$ for $2 \mathrm{~h}$ in an ambient air atmosphere.

\section{Characterization}

The structural studies were carried out using Philips model PW 1390 diffractometer at $\lambda=$ $1.5418 \AA$. The surface morphology of the sintered pellets was determined by JEOL scanning electron microscope. The nitrogen adsorption-desorption isotherms of the compounds were measured using a micrometrics adsorption instrument. The specific surface area $\left(\mathrm{m}^{2} / \mathrm{g}\right)$ of the composites was calculated using BET equation. FT-IR spectra were recorded on a Perkin-Elmer Fourier transform infrared spectroscopy. 


\section{Humidity measurements}

The $d c$ electrical resistance in different relative humidity $(\mathrm{RH} \%)$ for the compound in the form of pellets was determined by two-probe method. The change in surface conductivity as a function of applied field was measured using picoammeter (Keithley-6485). The controlled humidity environments were achieved using anhydrous $\mathrm{P}_{2} \mathrm{O}_{5}$ and saturated aqueous solution of $\mathrm{CH}_{3} \mathrm{COOK}, \quad \mathrm{CaCl}_{2} \cdot 2 \mathrm{H}_{2} \mathrm{O}$, $\mathrm{Ca}\left(\mathrm{NO}_{3}\right)_{2} \cdot 4 \mathrm{H}_{2} \mathrm{O}, \mathrm{NH}_{4} \mathrm{Cl}$ and $\mathrm{CuSO}_{4} .5 \mathrm{H}_{2} \mathrm{O}$ in a closed glass vessel at $298 \mathrm{~K}$ yielded $5,20 \%$, $31 \%, 51 \%, 79 \%$ and $98 \%$, relative humidity $(\mathrm{RH})$ respectively. The sensitivity factor $S_{f}$ was calculated by the ratio of resistances, $R_{5 \%} / R_{98 \%}$, where $R_{5 \%}$ and $R_{98 \%}$ are the $d c$ resistances at 5 and $98 \% \mathrm{RH}$, respectively. Response and recovery times were measured by using a degassed glass chamber.

The temperature-dependent electrical conductance experiments were carried out to determine the activation energy of the samples using the linearized form of the expression $I=$ $I_{0} \exp ^{-E a / k T}$, where $I$ is the current, $E_{a}$ the activation energy, $k$ the Boltzmann constant and $T$ the temperature $\left({ }^{\circ} \mathrm{C}\right)$.

\section{Results and Discussion}

FT-IR spectra (Fig. 1) of annealed bismuth iron molybdate (BFM -1) at $700^{\circ} \mathrm{C}$ showed sharp bands at $879,804,733,535$ (shoulder) and 450 $\mathrm{cm}^{-1}$. These values correspond to metal to oxygen stretching frequencies. The $\mathrm{v}(\mathrm{Mo}=\mathrm{O})$ symmetric strectching occur at $804.29 \mathrm{~cm}^{-1}$. Asymmetric $\mathrm{v}(\mathrm{Mo}-\mathrm{O}-\mathrm{Fe})$ band occurs at $733.38 \mathrm{~cm}^{-1}$. Symmertic band of $\mathrm{v}(\mathrm{Mo}-\mathrm{O}-\mathrm{Fe})$ band occurs at $535.22 \mathrm{~cm}^{-1}$.

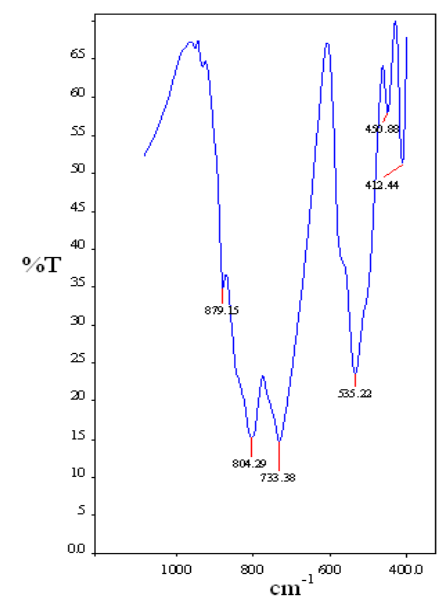

Fig. 1. FT-IR spectrum of $\mathrm{Bi}_{3} \mathrm{FeMo}_{2} \mathrm{O}_{12}(\mathrm{BFM}-1)$ sintered at $700^{\circ} \mathrm{C}$

The XRD patterns of BFM-1, BFM-2 and BFM-3 were shown in Fig.2(a-c). The $2 \theta$ values of $\mathrm{Bi}_{3} \mathrm{FeMo}_{2} \mathrm{O}_{12}$ (Fig 2a) are quite in agreement with JCPDS No 27-0047. The presence of $\mathrm{Ag}_{2} \mathrm{O}_{3}$ in BFM-2 and BFM-3 was confirmed and the $2 \theta$ values agree with JCPDS No 40-0909. The SEM images (Fig. $3 a$ and $3 b$ ) of BFM-1 and BFM-2 composites depict the intergranular porous structure of the prepared composites qualitatively. From the SEM image it is evident that the silver oxide addition will reduce the grain size and has well arranged stacked of grain size and pores. The Fig.3b showed more porous than the Fig.3a.

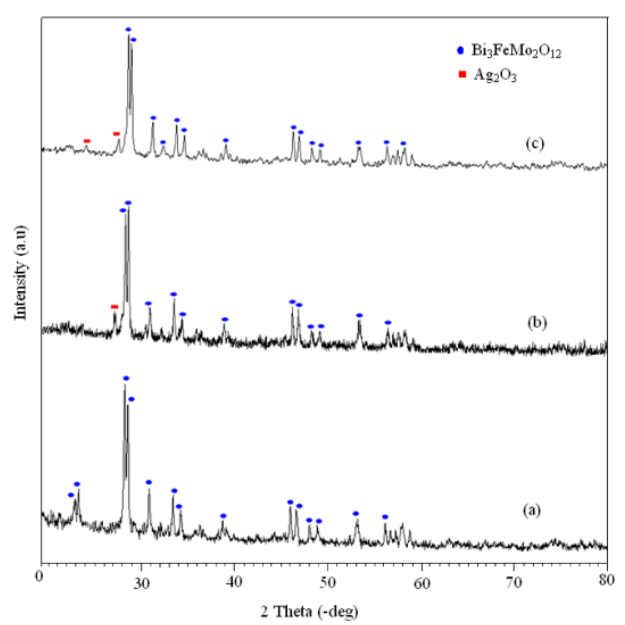

Fig. 2. X-ray diffraction spectra of (a) BFM-1, (b) $B F M-2$ and (c) BFM-3
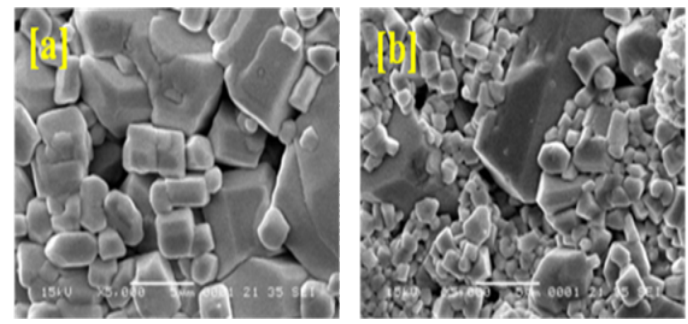

Fig. 3. SEM image of (a) BFM-1 and (b) BFM-2

The BET surface area of the compound BFM1, BFM-2 and BFM-3 was 8.81, 20.66 and $15.79 \mathrm{~m}^{2} / \mathrm{g}$ respectively. The increase in surface area for BFM-2 can be attributed to the decrease in grain size.

Humidity sensing property is a surface adsorption phenomenon. The resistance measurements as a function of $\mathrm{RH}$ at a fixed ambient temperature at $25^{\circ} \mathrm{C}$ are shown in Fig. 4. The plots of log $\mathrm{R}$ versus $\mathrm{RH} \%$ for all the BFM compounds are shown in Fig 4.

All the sensor samples studied showed a decrease in resistance values with increase in $\mathrm{RH}$, showing that the conduction occurred mainly at the grain surface, which was governed by the adsorbed water molecules $[8,9]$. The resistance of BFM-2 changed by 
almost four orders of magnitude between 5 and $98 \% \mathrm{RH}$. The decrease in log resistivity (log $\mathrm{R})$ is found maximum in BFM-2 indicating good intergrain connectivity as well as high surface area $\left(20.66 \mathrm{~m}^{2} / \mathrm{g}\right)$ due to high porosity resulting in easy chemisorption and physisorption of water vapours.

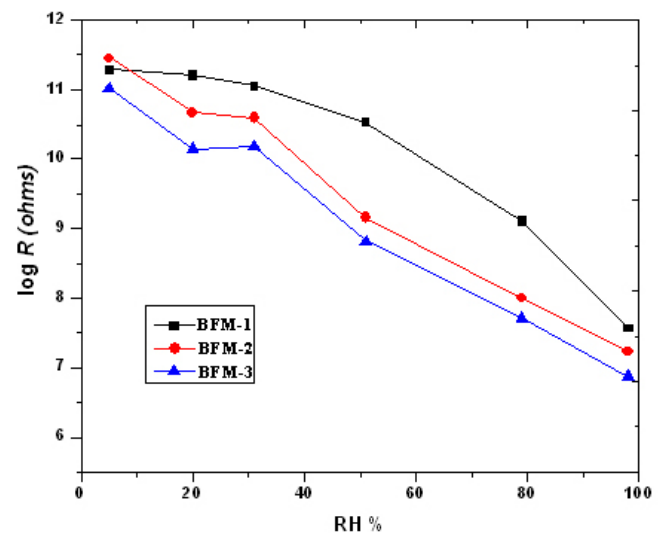

Fig. 4. Relative humidity vs. log R plots at ambient temperature

By adding silver oxide to bismuth iron molybdate, the porosity increased as shown by SEM (Fig. 3b). BFM-1 has less porosity when compared with BFM-2 compound. The sensitivity of BFM-1 is found to be $5177.4 \pm 180$. The BFM-1 has a pore size larger than the size of the water molecule and hence the adsorption of water and hydrogen bonding is less when compared with the other two compounds BFM2 and BFM-3. The BFM-2 shows the highest sensitivity factor towards the humidity, because it has more porosity when compared with BFM1 compound. Therefore, among the composites, the optimum amount of silver oxide to be added to obtain a high sensitivity factor is $5 \mathrm{~mol} \%(\mathrm{BFM}-2)$ and the sensitivity factor is about $16798.57 \pm 550$. After that, the sensitivity factor decreased, BFM-3 (10 mol\%) showed $13914.1 \pm 350$ towards humidity. Under low humidity, water adsorption on the sample surface is the dominant factor for electrical conduction. Therefore, a higher surface area would provide more sites for water adsorption and produce more charge carriers for electrical conduction.

Response and recovery times are the important parameters of good humidity sensors. The response and recovery characteristics were studied for the BFM-2 composite between 5 and $98 \% \mathrm{RH}$. The response and recovery time of BFM-2 were 260 and 180s, respectively.

The stability of BFM-2 compound was continuously monitored at $98 \% \mathrm{RH}$ for 30 days. It was found that the resistance was stable except for a small variation from $0.01 \times 10^{7}$ to
$0.03 \times 10^{7}$ ohms. This indicates the good stability and durability of the BFM-2 compound.

\section{Conclusion}

The bismuth iron molybdate (BFM-1) was prepared by co-precipitation method. 5 and 10 $\mathrm{mol} \%$ of silver nitrate was added to BFM-1 compound. The BFM-1, BFM-2 and BFM-3 were made into pellets and heated at $700^{\circ} \mathrm{C}$ for $2 \mathrm{~h}$ and studied for humidity sensing behaviour. The studies revealed that BFM-2 has grains of even size and open pores with good connectivity and hence acts as humidity sensor, with a good sensitivity factor (16798.57 \pm 550$)$.

\section{Acknowledgements}

The authors acknowledge the funding from UGC-Major Research Project 32-249/2006 (SR).

\section{References}

[1] Suman Pokhrel, B. Jeyaraj, K.S. Nagaraja, Material Letters 57, 3543-3548 (2003); doi: 10.1016/S0167-577X(03)00122-8

[2] M.K. Jain, M.C. Bhatnagar, G.L. Sharma, Sensors and Actuators B: Chemical 55, 180185 (1999); doi:10.1016/S0925-4005(99)000477

[3] A.M. Edwin Suresh Raj, C. Mallica, O.M. Sridharan, K.S. Nagaraja, Material Letters 53, 316-320 (2002); doi:10.1016/S0167$577 \times(01) 00499-2$

[4] C. Corrado, P. Mustarelli, A. Magistris, Journal of Solid State Chemistry 140, 91-96 (1998); doi: 10.1006/jssc.1998.7867.

[5] W. M. Sears, S. M. Mclntyre, Journal of applied physics 79, 7703-7707 (1996); doi: 10.1063/1.362435

[6] W. M. Sears, Sensors and Actuators B: Chemical 12, 95-102 (1993); doi: 10.1016/0925-4005(93)80004-U

[7] W. M. Sears, Langmuir 17, 5237-5244. (2001); doi: 10.1021/la001005c

[8] R. Sundaram, E.S. Raj, K.S. Nagaraja, Sensors and Actuators B: Chemical 99, 350354 (2004); doi: 10.1016/j.snb.2003.12.001

[9] R. Sundaram, K.S. Nagaraja, Sensors and Actuators B: Chemical 101, 353-360 (2004); doi: 10.1016/j.snb.2004.04.005. 